\title{
Cyclic Voltammetric and Electrical Impedance Spectroscopy Studies of Graphene- and Conductive Polymer-based Enzyme Electrodes
}

\author{
Nur Alya Batrisya Ismail, Nurul Izzati Ramli, Firdaus Abd-Wahab, \\ Wan Wardatul Amani Wan Salim*
}

Department of Biotechnology Engineering, Kulliyyah of Engineering,

International Islamic University Malaysia, 50728 Gombak, Kuala Lumpur, Malaysia

*Corresponding Author

DOI: https://doi.org/10.30880/ijie.2019.11.03.008

Received 17 March 2019; Accepted 4 July 2019; Available online 3 September 2019

\begin{abstract}
Screen-printed carbon electrodes (SPCEs), modified with graphene oxide and poly(3,4ethylenedioxythiophene):polystyrenesulfonic acid (GO-PEDOT:PSS/SPCEs), and SPCEs modified with partially reduced GO and PEDOT:PSS (prGO-PEDOT:PSS/SPCEs) were studied for electrochemical transduction. Randles Sevcik analysis showed that the prGO-PEDOT:PSS/SPCE has a higher effective surface area of $219.3 \mu \mathrm{m}^{2}$ in comparison to the unreduced GO-PEDOT:PSS/SPCE $\left(87.0 \mu \mathrm{m}^{2}\right)$ and the bare SPCE $\left(71.7 \mu \mathrm{m}^{2}\right)$. Using electrical impedance spectroscopy (EIS), we determined that the prGO-PEDOT:PSS/SPCE has a lower charge-transfer resistance $\left(R_{c t}\right)$ of $163.82 \Omega$ in comparis on to the GO-PEDOT:PSS/SPCE (427.87 $\Omega$ ) and the bare SPCE (13.31 k $\Omega$ ). Glucose oxidase (GOx) was immobilized on all electrode types, including GO/SPCE as additional control and tested with low $(0.2 \mathrm{mM})$, intermediate $(0.6 \mathrm{mM})$, and high $(1 \mathrm{mM})$ glucose concentrations. GOx/GO/SPCEs showed the largest change in anodic peak current $\left(\mathrm{I}_{\mathrm{pa}}\right), 8.5,7.5$ and $4.9 \mu \mathrm{A}$ for low, intermediate, and high glucose concentrations, respectively. Interestingly, GOx/prGO-PEDOT:PSS/SPCEs have no change in both anodic and cathodic peak current, although they exhibit better redox capability, while GOx/SPCEs have very low $\mathrm{I}_{\mathrm{pa}}$. The results show that the high effective surface area and low charge-transfer resistance $\left(\mathrm{R}_{\mathrm{ct}}\right)$ of prGO-PEDOT:PSS/SPCEs do not necessarily result in a sensitive glucose sensor in cases where immobilization of enzymes on the material can affect electron transfer.
\end{abstract}

Keywords: Cyclic voltammetry, electrical impedance spectroscopy, PEDOT:PSS, partially reduced graphene oxide, glucose oxidase, electrochemical transduction.

\section{Introduction}

Modifying electrodes by incorporating nanomaterials to enhance signal transduction is becoming a common approach in the field of electrochemical sensors. This trend is driven by ease and cost effectiveness, allowing for rapid fabrication of improved electrodes. The advent of graphene and conductive polymers has further facilitated developments in electrode modification. Numerous instances of the use of graphene and conducting polymers as composites in the development of biosens or [1-4], solar cells [5-7], and energy-storage applications [8-11] have been reported. Conductive polymer poly(3,4-ethylenedioxythiophene):polystyrenesulfonic acid, PEDOT:PSS, is the usualpolymer of choice in the fabrication, owing to its flexibility, stability, and cost effectiveness [12]. 
The graphene-PEDOT:PSS composite exploits the synergistic properties of both graphene and PEDOT:PSS, with the former having large surface area $\left(736.6 \mathrm{~m}^{2} / \mathrm{g}\right)$ [13] and abundant carboxylated functional groups [14,15] for biomolecule immobilization, and the latter high conductivity $(300 \mathrm{~S} / \mathrm{cm})$ and good electrocatalytic propert ies for electron transfer [16]. The increased use of the nanocomposite for biosensing purposes stems from a facile fabrication process that has potential for rapid prototyping of devices [17].

In this study, we explored the potential of drop-casting graphene and PEDOT:PSS on screen-printed carbon electrodes (SPCEs) with the intent for rapid prototyping. Although the use of graphene and PEDOT:PSS is not new for biosensors, most studies do not focus on or emphasize methods that are cost-effective with simple fabrication possibilities. The effect of these materials in either single or composite form on SPCEs is not well understood. Furthermore, when PEDOT:PSS was used, most studies looked into applications for solar cells [6,7,11,18-20]. For fabrication of reduced graphene and PEDOT:PSS on SPCEs, we drop-cast a solution containing graphene oxide (GO) and PEDOT:PSS on SPCEs, electrochemically reduced the GO to yield a partially reduced graphene and PEDOT:PSS nanocomposite (prGO-PEDOT:PSS/SPCE), and immobilized the model enzyme glucose oxidase (GOx) for glucose sensing (GOx/prGO-PEDOT:PSS/SPCE). The fabrication process does not require a cleanroom facility and was done in ambient conditions.

Partially reduced graphene (prGO) is produced by mild electrochemical reduction of GO in a slightly acidic buffer solution ( $\mathrm{pH}$ ) to prevent the loss of all GO oxygenated functional groups [21-24]. For prGO, the $\pi$ conjugation is partially restored, while the conductivity of the material is maintained [25]. Electrochemical reduction of GO to prGO is a safe and environmentally friendly process in comparison to thermal and chemical reduction [26].

Electrochemical analysis by cyclic voltammetry (CV) and electrochemical impedance spectroscopy (EIS) was conducted on SPCEs, GO-PEDOT:PSS/SPCEs, and prGO-PEDOT:PSS/SPCEs to investigate the feasibility of the materials as transducers. In CV, we look into the current versus square root of scan rate and into the effective surface area to reveal electrode electrocatalytic activity and electron-transfer reversibility, while in EIS, we look into the chargetransfer resistance (Rct) at the electrode-solution interface [27,28]. We report on the aforementioned parameters from CV and EIS to identify the potential of the prGO-PEDOT:PSS as an ion-to-electron transducer, with SPCEs and GOPEDOT:PSS/SPCEs as controls.

In addition to the choice of materials for transducers, enzyme immobilization on the transducer surface can affect sensitivity and limit of detection. We have immobilized glucose oxidase on electrodes through methods of physical adsorption [29-31]. However, adsorption can result in enzyme leaching, reducing the sensitivity of detection. Immobilization of glucose oxidase with glutaraldehyde as crosslinker proved successfulfor glucose sensing [1,32-34]; such crosslinking provides a stable template for enzyme immobilization and prevents enzyme leaching [34-37]. A solid immobilization strategy exposes the active enzyme site to the analyte and retains biological activity, resulting in highly sensitive biosensors. Therefore, we immobilized GOx through glutaraldehyde crosslinking on prGOPEDOT:PSS/SPCEs, and controls of SPCEs, GO/SPCEs and GO-PEDOT:PSS/SPCEs.

We fabricated and tested the functionality of prGO-PEDOT:PSS/SPCEs and controls of SPCEs, GO/SPCEs and GOPEDOT:PSS/SPCEs at low $(0.2 \mathrm{mM})$, intermediate $(0.6 \mathrm{mM})$, and high $(1 \mathrm{mM})$ glucose concentration for glucose sensing. In glucose sensing, GOx is exposed to glucose to form an enzyme-analyte complex. The active site of an enzyme is reduced by accepting electrons at the transducer, where the signal is measured by a potentiostat (Figure 1). Although many studies have reported on highly sensitive biosensors, we believe that our facile fabrication method with no requirement for any cleanroom facility can lead to commercialization of electrochemical biodevices for noninvasive glucose sensing. This study will report on the fabrication process, electrochemical characterization of the transducer, and sensing of glucose. 


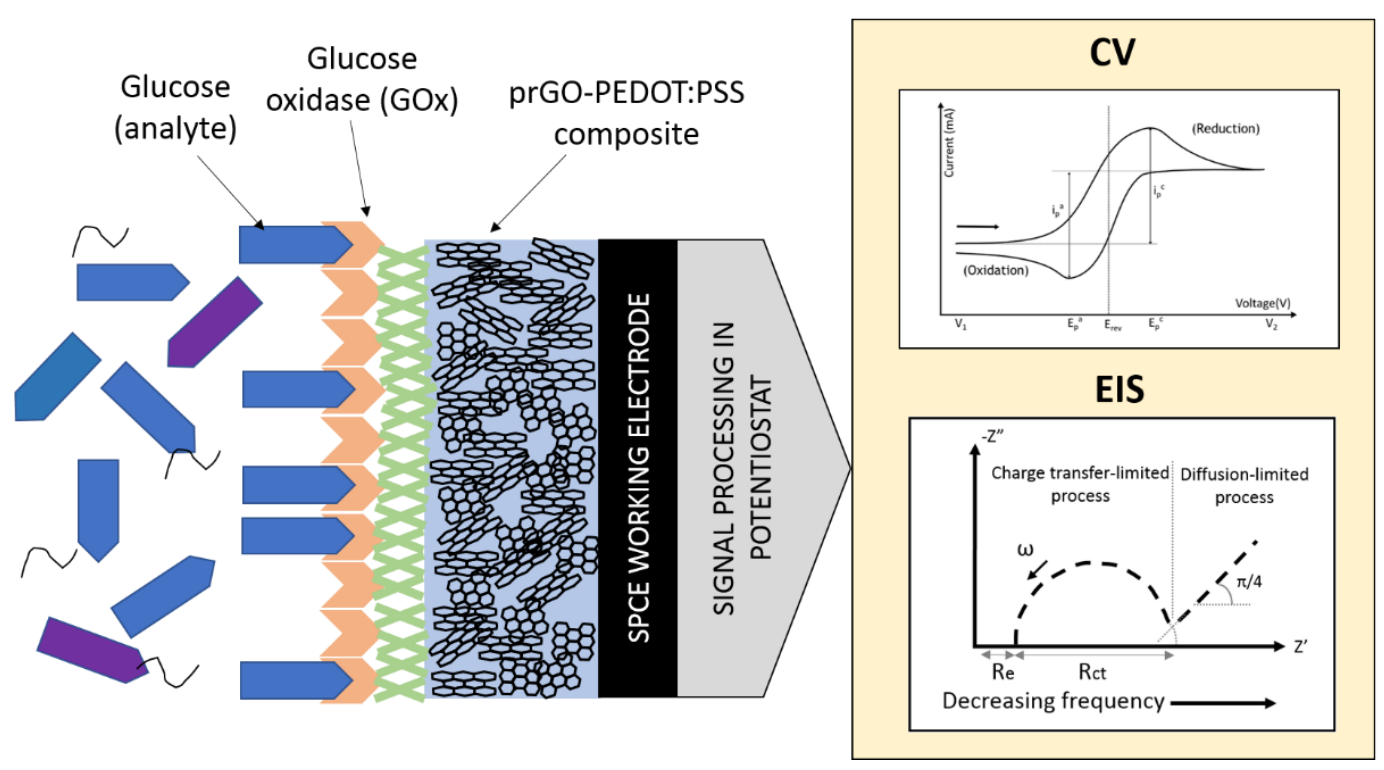

Fig. 1 - Detection mechanism for glucose biosensing. The glucose oxidase (GOx) immobilized on the electrode is exposed to glucose and forms an enzyme-analyte complex that is detected by cyclic voltammetry (CV) and electrochemical impedance spectroscopy (EIS). Electrons successfully transduced through the electrode surface are reported as current (I) against voltage (V). For successful detection, the electrons must shuttle through the enzyme-analyte layer. EIS measures resistive behavior against electrochemical reaction at the electrode-solution interface where the real $\left(Z_{r e}\right)$ and imaginary $\left(Z_{i m}\right)$ impedances are obtained.

\section{Materials and Methods}

\subsection{Chemicals and reagents}

Chemicals used in this work were analytical grade and is described in [40]. For glucose measurements, glutaraldehyde, glucose oxidase (GOx, 15,000 units/g solid), and D-(+)-glucose $\left(\mathrm{C}_{6} \mathrm{H}_{12} \mathrm{O}_{6}\right)$ were purchased from SigmaAldrich, St. Louis, Mis souri, USA.

\subsection{Fabrication of nanomaterial-modified screen-printed carbon electrodes for glucose sensing}

The fabrication steps for a glucose oxidase/partially reduced graphene oxide/poly(3,4ethylenedioxythiophene):polystyrenesulfonic acid/screen-printed carbon electrode (SPCE), denoted as GOx/prGOPEDOT:PSS/SPCE, are shown in Figure 2. Fabrication of GO/SPCEs and GO/PEDOT:PSS/SPCEs follows a similar process of drop-casting; SPCEs are unmodified electrodes.

Pretreatment of the SPCEs was conducted via cyclic voltammetry (CV) for $3 \mathrm{CV}$ cycles in $0.1 \mathrm{~N} \mathrm{H}_{2} \mathrm{SO}_{4}$ at a scan rate of $100 \mathrm{mV} / \mathrm{s}$ and potential range of -2.5 to $2.5 \mathrm{~V}$ [4]. Activation of SPCEs with anodic and cathodic potential increases the porosity of the electrode surface, improves voltammetry responses, and removes adsorbed oxygen [38,39]. After pretreatment, the electrodes were rinsed with DI water and dried at ambient temperature. The method for GOPEDOT:PSS composite preparation, deposition, and reduction of GO-PEDOT:PSS/SPCEs to prGO-PEDOT:PSS/SPCEs were reported previously [40,41]. The reduced prGO-PEDOT:PSS/SPCEs were rinsed with PBS, $\mathrm{pH} 7.4$, and dried in ambient conditions.

Fifty $\mu \mathrm{l}$ glucose oxidase (GOx) solution $(1 \mathrm{mg} / \mathrm{ml})$ was mixed with $20 \mu \mathrm{l}$ of $2 \%$ (vol) glutaraldehyde [1] to form GOx-glutaraldehyde solution. Three $\mu$ s solution was drop-cast onto SPCEs, GO-PEDOT:PSS/SPCEs, and prGOPEDOT:PSS/SPCEs to form GOx/SPCEs, GOx/GO-PEDOT:PSS/SPCEs, in and GOx/prGO-PEDOT:PSS/SPCEs. The fabricated electrodes were dried for 24 hours in ambient conditions before further measurements. For the purpose of glucose detection, a $20 \mathrm{mM}$ glucose stock solution was prepared by dissolving $0.36 \mathrm{~g} \mathrm{D}-(+)$-glucose powder in $100 \mathrm{ml}$ of $0.01 \mathrm{M}$ PBS, $\mathrm{pH}$ 7.4. Glucose solutions of $0.2,0.6$, and $1 \mathrm{mM}$ were prepared by serial dilution of the prepared stock solution to the desired concentration. Electrochemical analysis of the modified electrodes was performed using a pocketSTAT (IVIUM Technologies, Eindhoven, The Netherlands). 


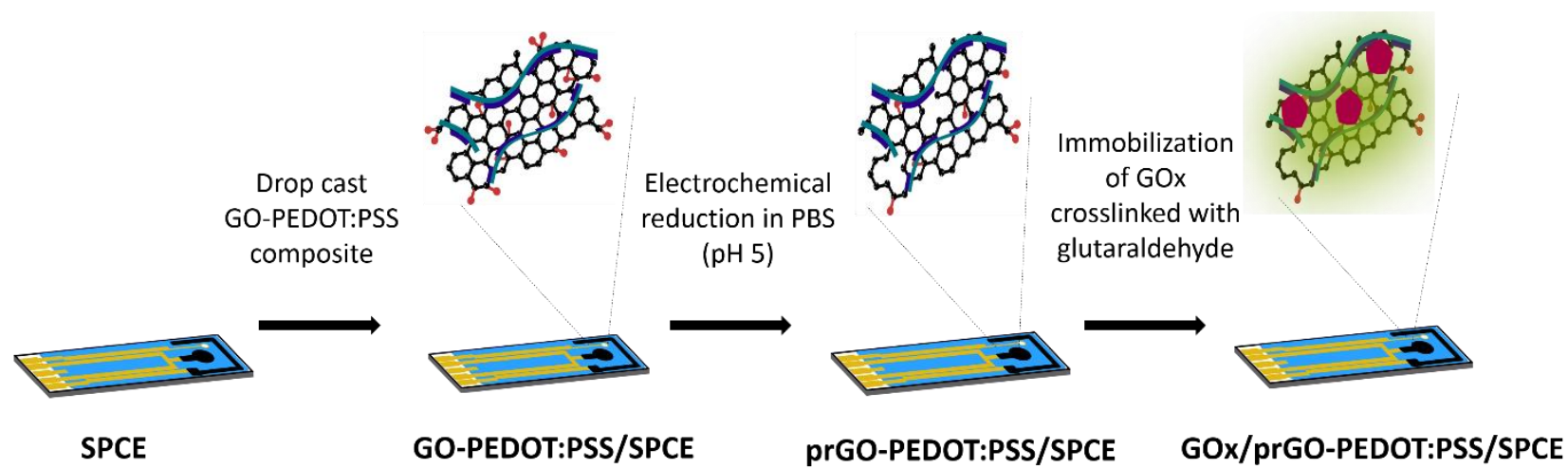

Fig. 2 - Fabrication steps of prGO-PEDOT:PSS/SPCEs for glucose sensing. A screen-printed carbon electrode (SPCE) activated through CV was used as a conductive substrate. Modification of the SPCE was done by dropcasting $3 \mu \mathrm{l}$ GO-PEDOT:PSS composite onto the working electrode. Next, the electrical conductivity of GO was restored by electrochemical reduction in PBS, pH 5, which results in the formation of a partially reduced graphene oxide-PEDOT:PSS (prGO-PEDOT:PSS) layer on the SPCE Subsequent immobilization of glucose oxidase (GOx) was accomplished through crosslinking with glutaraldehyde [40,41] on the prGO-PEDOT:PSS/SPCE.

\subsection{Cyclic voltammetry and electrical impedance spectroscopy}

$\mathrm{CV}$ and electrical impedance spectroscopy measurements is described in [40]. For glucose detection, CVs were carried out in 0.01 M PBS, pH 7.4, at a scanning potential of -0.5 to $1 \mathrm{~V}$ for GOx/SPCEs, GOx/GO/SPCEs, GOx/GOPEDOT:PSS/SPCEs, and GOx/prGO-PEDOT:PSS/SPCEs. These electrodes measured glucose at low (0.2 mM), intermediate $(0.6 \mathrm{mM})$, and high $(1 \mathrm{mM})$ concentrations (Figure 6) at $\mathrm{pH} 7.4$.

\subsection{Statistical analysis}

Statistical analysis conducted in this work is described [40].

\section{Results and Discussion}

\subsection{Cyclic voltammetry for different electrode modifications}

Figure 3 shows the CV measurements on SPCEs, GO-PEDOT:PSS/SPCEs, and prGO-PEDOT:PSS/SPCEs using $0.1 \mathrm{M} \mathrm{K}_{3}\left[\mathrm{Fe}(\mathrm{CN})_{6}\right]$ as the redox mediator. Linear regression plots of peak current $\left(\mathrm{I}_{\mathrm{p}}\right)$ against the square root of scan rate $\left(\mathrm{v}^{1 / 2}\right)$ suggested that electron transfer at the electrode-electrolyte interface is reversible for all electrodes. The scan rates used for this study are 25, 50,100, 150, and $200 \mathrm{mV} / \mathrm{s}$. As can be seen in figure 3(b), there exists a shift in peak potential $\left(E_{p}\right)$ of GO-PEDOT:PSS/SPCEs, suggesting sluggish electron transfer. The linear regression plots of each electrode modification also imply the dependence of $\mathrm{I}_{\mathrm{p}}$ on scan rate, which can be described by the Randles -Sevcik equation [3] [42].

Reversibility of electron transfer ensures that the analyte can undergo a repeatable redox process. A forward scan from $-0.5 \mathrm{~V}$ to $1 \mathrm{~V}$ oxidizes $\mathrm{Fe}(\mathrm{CN}) 6^{-4}$ and produces the anodic current. A reverse scan from $1 \mathrm{~V}$ back to the initial potential of $-0.5 \mathrm{~V}$ reduces $\mathrm{Fe}(\mathrm{CN}) 6^{-3}$ to $\mathrm{Fe}(\mathrm{CN}) 6^{-4}$ and produces the cathodic current. 
(a)

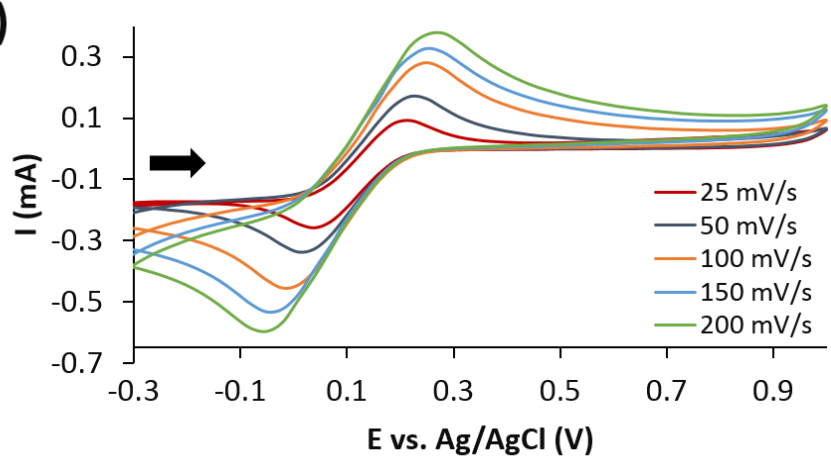

(b)

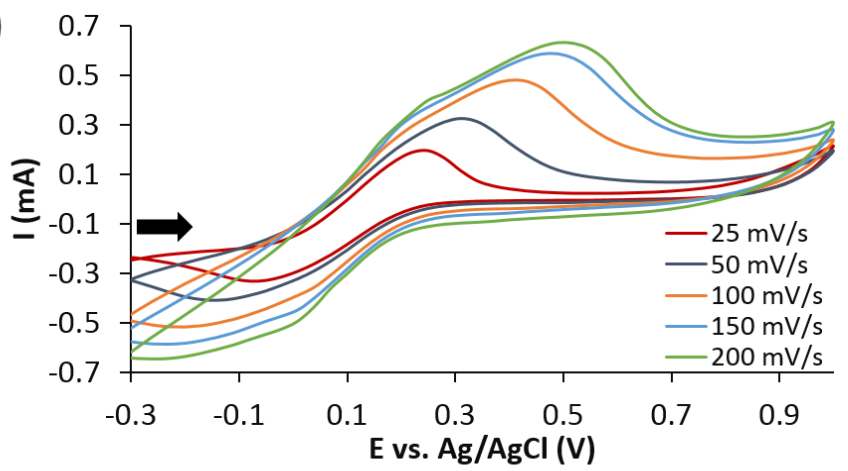

(c)

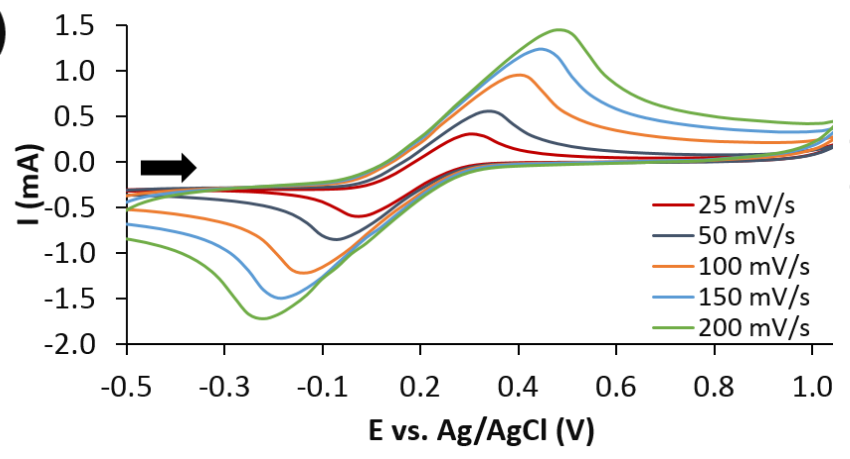

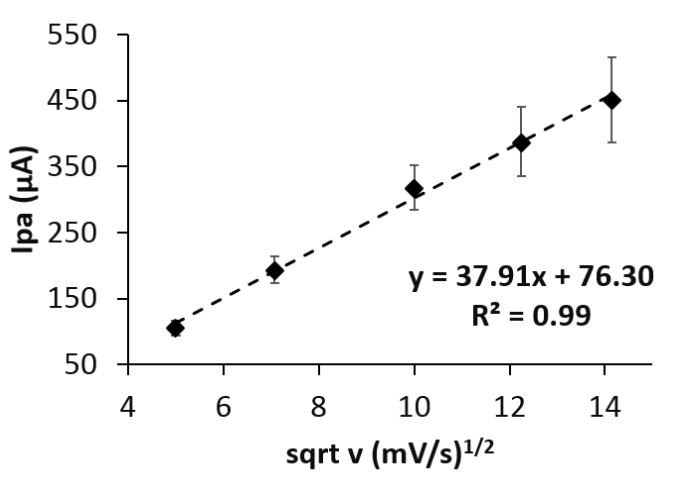
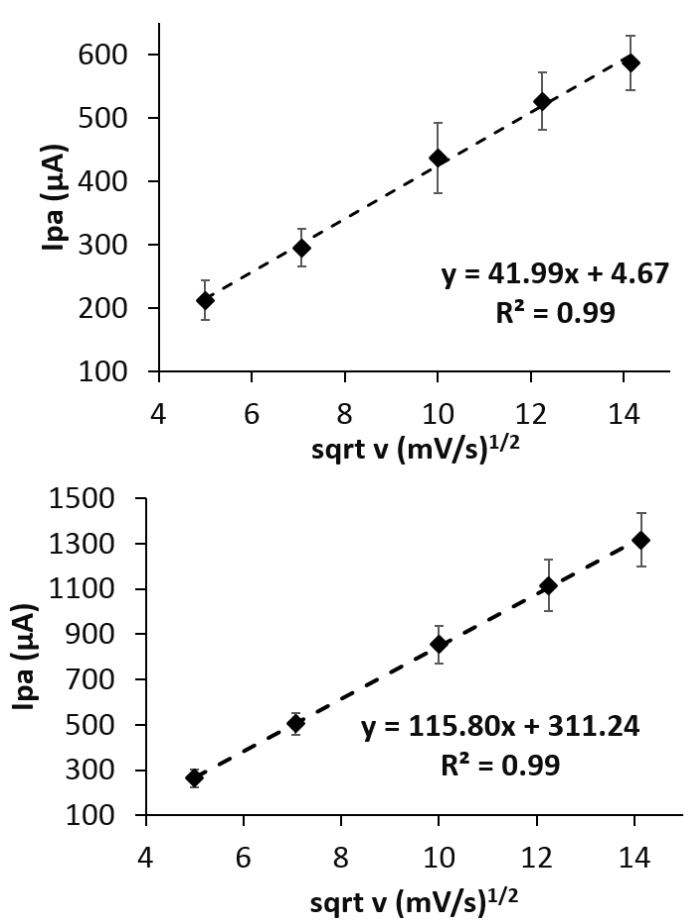

Fig. 3 - Cyclic voltammograms (CVs) of SPCEs (a), GO-PEDOT:PSS/SPCEs (b), and prGO-PEDOT:PSS/SPCEs (c) in $0.1 \mathrm{M} \mathrm{K}_{3}\left[\mathrm{Fe}(\mathrm{CN})_{6}\right]$ at scan rates of $25,50,100,150$ and $200 \mathrm{mV} / \mathrm{s}$ with corresponding linear regression between the peak current $\left(I_{p}\right)$ and the square root of the scan rate $\left(v^{1 / 2}\right)$. The slope $(k)$ of the linear plot is used to calculate the electroactive surface area of SPCEs, GO-PEDOT:PSS/SPCEs, and prGO-PEDOT:PSS/SPCE using the Randles-Sevcik equation as reported in [40].

Figure 4 shows CVs of SPCEs, GO-PEDOT:PSS/SPCEs, and prGO-PEDOT:PSS/SPCEs at a scan rate of $100 \mathrm{mV} / \mathrm{s}$. The anodic and cathodic peak potentials, respectively, are $\mathrm{E}_{\mathrm{pa}}=0.25 \mathrm{~V}$ and $\mathrm{E}_{\mathrm{pc}}=-0.02 \mathrm{~V}$ for bare SPCEs, $\mathrm{E}_{\mathrm{pa}}=0.40 \mathrm{~V}$ and $\mathrm{E}_{\mathrm{pc}}=-0.2 \mathrm{~V}$ for GO-PEDOT:PSS/SPCEs, $\mathrm{E}_{\mathrm{pa}}=0.34 \mathrm{~V}$ and $\mathrm{E}_{\mathrm{pc}}=-0.08 \mathrm{~V}$ for prGO-PEDOT:PSS/SPCEs. The peakto-peak potential difference $\left(\Delta \mathrm{E}_{\mathrm{p}}=\mathrm{E}_{\mathrm{pa}}-\mathrm{E}_{\mathrm{pc}}\right)$ of SPCEs is $0.27 \mathrm{~V}$, while $\Delta \mathrm{E}_{\mathrm{p}}$ for GO-PEDOT:PSS/SPCEs and prGOPEDOT:PSS/SPCEs is $0.42 \mathrm{~V}$. The similar $\Delta \mathrm{E}_{\mathrm{p}}$ for GO-PEDOT:PSS/SPCEs and prGO-PEDOT:PSS/SPCEs suggests that the nanocomposites shifted the peak potential without altering the electrode surface.

The anodic peak current $\left(\mathrm{I}_{\mathrm{pa}}\right)$ for SPCEs, GO-PEDOT:PSS/SPCEs, and prGO-PEDOT:PSS/SPCEs is $318.3 \mu \mathrm{A}$, $428.7 \mu \mathrm{A}$, and $856.3 \mu \mathrm{A}$, respectively. The electroactive surface area for SPCEs, GO-PEDOT:PSS/SPCEs, and prGOPEDOT:PSS/SPCEs is $71.7 \mu \mathrm{m}^{2}, 87.0 \mu \mathrm{m}^{2}$, and $219.3 \mu \mathrm{m}^{2}$, respectively. The increase in the electroactive surface area of prGO-PEDOT:PSS/SPCEs from GO-PEDOT:PSS/SPCEs could be due to the reduction of GO to prGO. The structure of prGO is made up of some oxygenated functional groups on the edges and basal plane of carbon atoms [15,22]. Furthermore, $\mathrm{prGO}$ has a semi-restored $\pi$ conjugation and $\mathrm{sp}^{2}$ lattice, which increases the conductivity of the material compared to GO [25]. 
PEDOT:PSS has excellent stability in aqueous media [43], high conductivity (1 S/cm), and transparency. Furthermore, PEDOT:PSS was found to retain its electrocatalytic activity even after electrochemical reduction [44] which explains the high peak current in prGO-PEDOT:PSS/SPCEs $(856.3 \mu \mathrm{A})$. When combining with graphene, PEDOT:PSS could inhibit the stacking of the graphene layer [4]. The synergistic interaction of prGO and PEDOT:PSS could assist the electron transfer between the redox analyte and the electrodes interface. The aforementioned properties indicate that prGO-PEDOT:PSS composite could be useful as a solid-state transducer for glucose sensing.

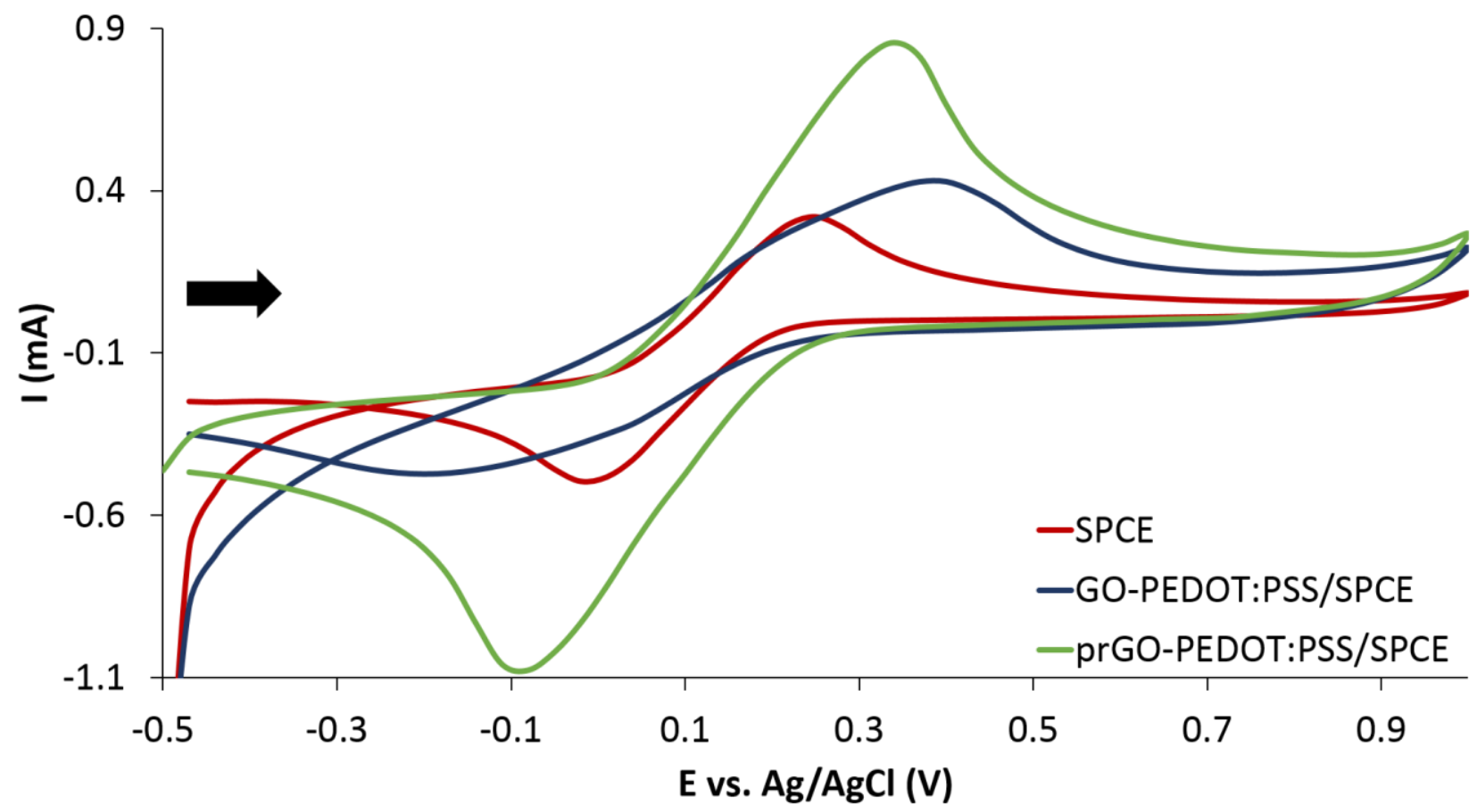

Fig. 4 - Cyclic voltammograms (CV) of SPCEs, GO-PEDOT:PSS/SPCEs, and prGO-PEDOT:PSS/SPCEs at a scan rate of $100 \mathrm{mV} / \mathrm{s}$ and potential range of -0.5 to $1 \mathrm{~V}$ in $0.1 \mathrm{M} \mathrm{K}$ [ $\left[\mathrm{Fe}(\mathrm{CN})_{6}\right]$. The anodic peak current $\left(\mathrm{I}_{\mathrm{pa}}\right)$ of the SPCEs, GO-PEDOT:PSS/SPCEs, and prGO-PEDOT:PSS/SPCEs was measured as $318.3 \mu \mathrm{A}, 428.7 \mu \mathrm{A}$ and 856.3 $\mu \mathrm{A}$, respectively. The shift in the anodic peak potential was observed and measured as $0.25 \mathrm{~V}, 0.40 \mathrm{~V}$ and $0.34 \mathrm{~V}$ for SPCEs, GO-PEDOT:PSS/SPCEs, and prGO-PEDOT:PSS/SPCEs, respectively.

\subsection{Electrical impedance spectroscopy for different electrode modifications}

Electrical impedance spectroscopy (EIS) was used to determine the charge-transfer resistance ( $\mathrm{R}_{\mathrm{ct}}$ ) for the electrodes. Figure 5 shows the Nyquist plot of SPCEs, GO-PEDOT:PSS/SPCEs, and prGO-PEDOT:PSS/SPCEs over a frequency range of $100 \mathrm{kHz}$ to $0.01 \mathrm{~Hz}$. The impedance spectroscopy was fitted to the Randles equivalent circuit (inset of figure 5a) using the IviumSoft ${ }^{\mathrm{TM}}$ electrochemistry software (https://www.ivium.com/software/). A typical Randles equivalent circuit is described in [45-48]. The Nyquist plot fitted with a Randles equivalent circuit was typically characterized by a $45^{\circ}$ linear region at lower frequency, which indicates a mass transfer-limited process. For an electron transfer-limited process, the semicircular region at high frequency of the Nyquist plot shows that $R_{c t}$ becomes dominant [49].

Figure $5 \mathrm{~b}$ shows the charge-transfer res istance for SPCEs, GO-PEDOT:PSS/SPCEs, and prGO-PEDOT:PSS/SPCE s to be $13.31 \mathrm{k} \Omega, 427.87 \Omega$ and $163.82 \Omega$, respectively. For unmodified SPCEs, a large semicircular shape is formed across the scanned frequency range. At high frequency, a small semicircular region followed by a linear region is observed in GO-PEDOT:PSS/SPCEs. The prGO-PEDOT:PSS/SPCE plot has a smaller semicircular region and a more prominent linear region, which suggests a mass transfer-limited process based on the Warburg impedance, $Z_{\mathrm{w}}[45-47,49-51]$.

The prGO-PEDOT:PSS/SPCE has the lowest $\mathrm{R}_{\mathrm{ct}}$, which is preferable for electron transfer and effective electrochemical transduction. Several studies have showed that PEDOT:PSS is a stable hole conductor that, when combined with GO, lowers $R_{c t}[6,52]$. A graphene oxide that is partially reduced was found to improve electron transfer at the electrode-solution interface [10]. For glucose biosensors, a prGO-PEDOT:PSS/SPCE can improve sensitivity and limit of detection. Immobilization of enzymes onto transducermaterials can increase $\mathrm{R}_{\mathrm{ct}}$ as a result of reduced electron transfer [50,53]. 
Statistically, the differences in $\mathrm{R}_{\mathrm{ct}}$ values for modified electrodes in comparison with an unmodified SPCE were highly significant, with adjusted $p$-values $<0.0001$. The differences in $\mathrm{R}_{\mathrm{ct}}$ for GO-PEDOT:PSS/SPCEs and prGOPEDOT:PSS/SPCEs were moderately significant, with $p$-values $<0.05$.
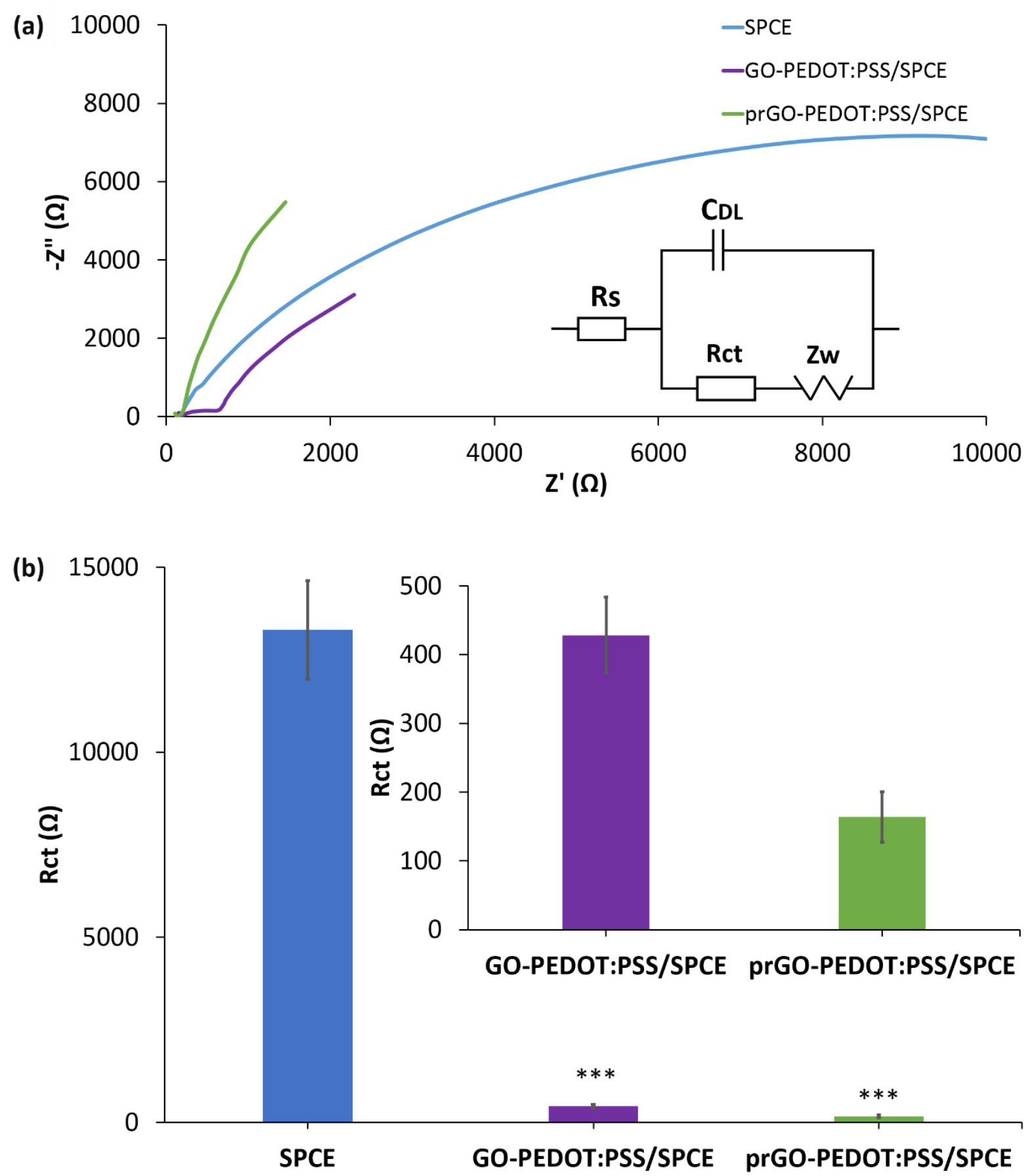

Fig. 5 - (a) Nyquist plot of SPCEs, GO-PEDOT:PSS/SPCEs, and prGO-PEDOT:PSS/SPCEs measured over a frequency range of $100 \mathrm{kHz}$ to $0.01 \mathrm{~Hz}$ with applied potential of $0.25 \mathrm{~V}$ and amplitude $10 \mathrm{mV}$. The electrochemical impedance spectroscopy was measured in $0.01 \mathrm{M}$ PBS, pH 7.4, containing $5 \mathrm{mM} \mathrm{K}_{3}\left[\mathrm{Fe}(\mathrm{CN})_{6}\right] / \mathrm{K}_{4}\left[\mathrm{Fe}(\mathrm{CN})_{6}\right](1: 1)$ as redox probe. Inset shows Randles equivalent circuit that was used to fit the enzyme-based electrochemical biosensor. (b) Graph of charge-transfer resistance for SPCEs, GO-PEDOT:PSS/SPCEs, and prGOPEDOT:PSS/SPCEs. Statistical analysis in comparison with SPCEs shows *** for $p<0.001(\mathrm{n}=3)$. 


\subsection{Cyclic voltammetry of different electrode modifications for glucose detection}

Electrochemical signals at the electrode-solution interface for glucose detection are dependent on the surface characteristics of the electrodes. To minimize the effect of adsorbed residualoxygen on the electrode surfaces, which can affect CV measurements, an electrode pretreatment process was conducted as described in section 2.2. As can be seen from figure 6, broad peaks with corresponding peak current $\left(\mathrm{I}_{\mathrm{p}}\right)$ exist for all electrodes except GOx/GOPEDOT:PSS/SPCEs, which have no identifiable anodic or cathodic peak current. Note also that these samples exhibit broader peaks because measurements are made in PBS, 7.4, and not in potassiumferricyanide $\left(\mathrm{K}_{3}\left[\mathrm{Fe}(\mathrm{CN})_{6}\right]\right)$. The broad anodic/cathodic peak current for GOx/SPCEs, GOx/GO/SPCEs, and GOx/prGO-PEDOT:PSS/SPCEs resulted from oxidation/reduction of oxygen in measurement solution, or an oxygen layer adsorbed on the electrode surfaces [54]. Any peaks observed could be the result of glucose oxidase catalyzing the glucose reaction with oxygen and producing gluconic acid and $\mathrm{H}_{2} \mathrm{O}_{2}$, where $\mathrm{H}_{2} \mathrm{O}_{2}$ can result in Faradaic signal. The shift in peaks for all electrodes could be the result of different mass- and charge transfer resistance $\left(\mathrm{R}_{\mathrm{ct}}\right)$ of the different materials used for enzyme immobilization [54]. Spiking the buffer solution with glucose at low $(0.2 \mathrm{mM})$, intermediate $(0.6 \mathrm{mM})$, and high $(1.0 \mathrm{mM}) \mathrm{glucose}$ concentrations does not result in obvious change in $\mathrm{I}_{\mathrm{p}}$ for GOx/SPCEs, GOx/GO/SPCEs, and GOx/prGOPEDOT:PSS/SPCEs, while GOx/GO/SPCEs resulted in the highest change in anodic and cathodic $\mathrm{I}_{\mathrm{p}}$ at the different glucose concentrations (Table 1). In a normal reaction, addition of glucose should decrease the peak current owing to the consumption of oxygen when glucose oxidase catalyzed the oxidation of glucose. Note here that only for every 2 moles of $\mathrm{H}_{2} \mathrm{O}_{2}$ resulted in 1 mole of oxygen, and oxygen will often leave the electrode surface through diffusion, which should result in lowering of the $\mathrm{I}_{\mathrm{p}}$. However, it seems that the resulting change is not obvious for GOx/prGOPEDOT:PSS/SPCEs. Larger changes in $\mathrm{I}_{\mathrm{p}}$ for GOx/GO/SPCEs could be the result of better glucose immobilizat ion at the electrode surfaces.

It is interesting to note here that the PEDOT:PSS samples retain $\mathrm{I}_{\mathrm{p}}$ values with addition of low, intermediate, and high glucose. This could be the result of PEDOT:PSS having a capacitive effect as a result of the electrical double layer at the interface of PEDOT and PSS nanoscale grains, where charges are retained on PEDOT:PSS instead of being measured by the SPCE [55]. The capacitive effect of PEDOT:PSS is further confirmed by the square-shaped CV curve as shown in figure 6. Reduced graphene oxide (rGO) was also found to be a good oxygen barrier owing to its multilayer structure and nano interlayer distance between the sheets [56]. The combination of both partially reduced graphene (prGO) and PEDOT:PSS in GOx/prGO-PEDOT:PSS/SPCEs could affect transport of oxygen at the electrode-solution interface. Further studies are needed to confirm this claim.

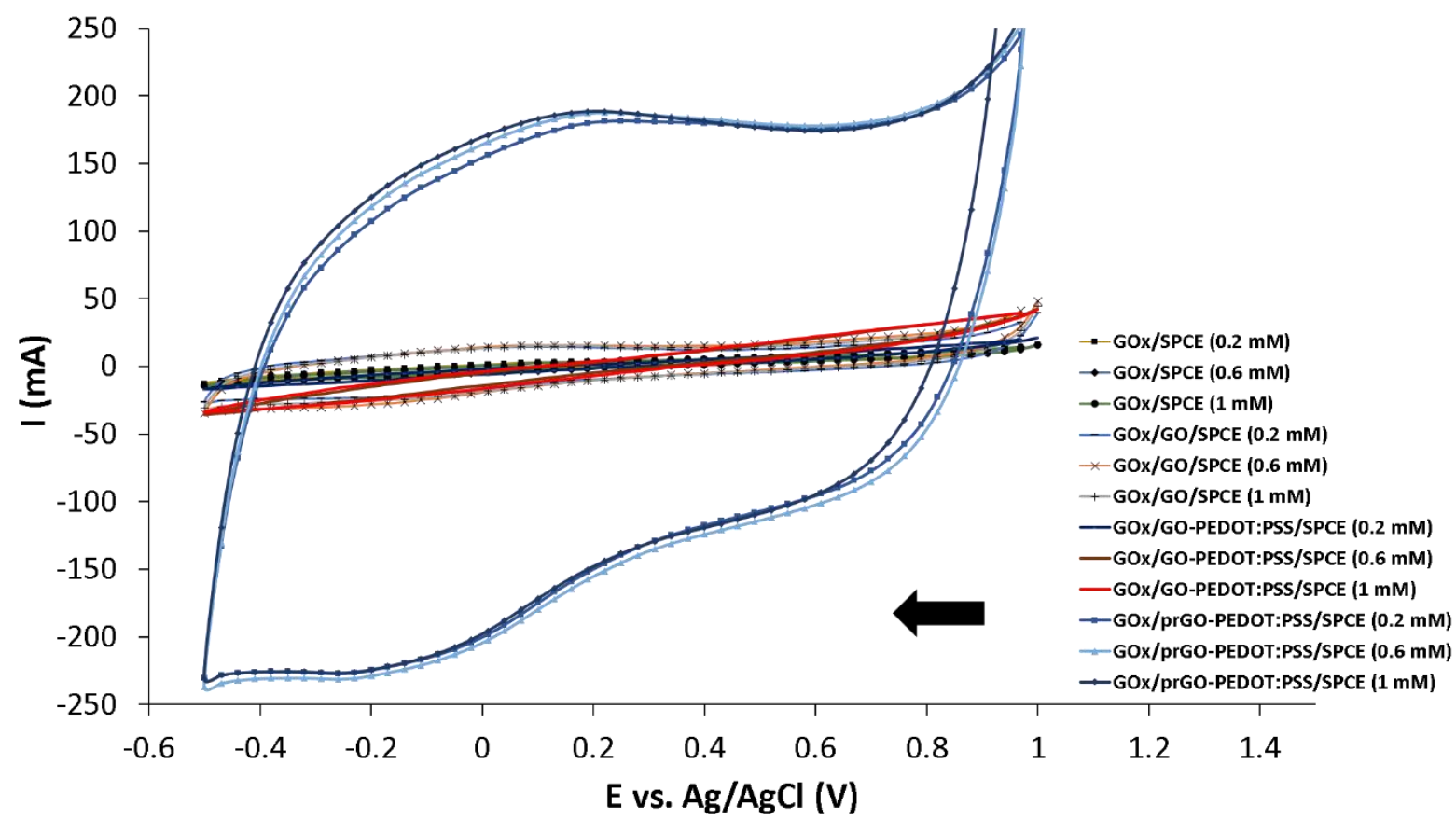

Fig. 6 - Cyclic voltammograms of GOx/SPCEs, GOx/GO/SPCEs, GOx/GO-PEDOT:PSS/SPCEs, and GOx/pr GO PEDOT:PSS/SPCEs in 0.01 M PBS, pH 7.4, with glucose concentrations of $0.2,0.6$ and $1.0 \mathrm{mM}$ at a scan rate of $100 \mathrm{mV} / \mathrm{s}$. Arrow corresponds to the direction of forward scan as reduction, and reverse scan as oxidation. 
Table 1 - Peak current of GOx/SPCEs, GOx/GO/SPCEs, and GOx/prGO-PEDOT:PSS/SPCEs at glucose concentrations of $0.2,0.6$ and $1.0 \mathrm{mM}$ at a scan rate of $100 \mathrm{mV} / \mathrm{s}$.

\begin{tabular}{|c|c|c|c|c|c|c|c|c|c|c|c|c|}
\hline \multirow{2}{*}{$\begin{array}{c}\text { Glucose } \\
\text { concentration } \\
(\mathbf{m M})\end{array}$} & \multicolumn{3}{|c|}{ GOX/SPCE } & \multicolumn{3}{|c|}{ GOx/GO/SPCE } & \multicolumn{6}{|c|}{ GOx/prGO-PEDOT:PSS/SPCE } \\
\hline & $\begin{array}{l}\mathbf{E}_{\mathbf{p a}} \\
(\mathbf{V})\end{array}$ & $\begin{array}{c}\mathbf{I}_{\mathbf{p a}} \\
(\boldsymbol{\mu A})\end{array}$ & $\begin{array}{l}\text { Std. } \\
\text { error }\end{array}$ & $\begin{array}{l}\mathbf{E}_{\text {pa }} \\
(\mathbf{V})\end{array}$ & $\begin{array}{c}\mathbf{I}_{\mathbf{p a}} \\
(\boldsymbol{\mu A})\end{array}$ & $\begin{array}{l}\text { Std. } \\
\text { error }\end{array}$ & $\begin{array}{l}\mathbf{E}_{\mathbf{p c}} \\
(\mathbf{V})\end{array}$ & $\begin{array}{c}\mathbf{I}_{\mathrm{pc}} \\
(\boldsymbol{\mu A})\end{array}$ & $\begin{array}{c}\text { Std } \\
\text { error }\end{array}$ & $\begin{array}{l}\mathbf{E}_{\mathbf{p a}} \\
(\mathbf{V})\end{array}$ & $\begin{array}{c}\mathbf{I}_{\mathrm{pa}} \\
(\boldsymbol{\mu} \mathbf{A})\end{array}$ & $\begin{array}{l}\text { Std. } \\
\text { error }\end{array}$ \\
\hline 0.2 & 0.08 & 0.000693 & 0.000415 & 0.09 & 8.58 & 0.558 & -0.26 & -227.38 & 3.686 & 0.07 & 181.48 & 0.446 \\
\hline 0.6 & 0.11 & 0.000519 & 0.000113 & 0.14 & 7.56 & 1.395 & -0.26 & -231.32 & 4.091 & 0.13 & 187.62 & 0.358 \\
\hline 1.0 & 0.10 & 0.000154 & 0.000135 & 0.10 & 4.93 & 0.373 & -0.26 & -226.56 & 2.229 & 0.10 & 187.72 & 0.406 \\
\hline
\end{tabular}

\section{Conclusion}

$\mathrm{CV}$ measurements in a redox solution of potassium ferricyanide $\left(\mathrm{K}_{3}\left[\mathrm{Fe}(\mathrm{CN})_{6}\right]\right)$ showed prGO-PEDOT:PSS/SPCEs to have the largest effective surface area of $219.3 \mu \mathrm{m}^{2}$ compared to SPCEs and GO-PEDOT:PSS/SPCEs. In EIS, prGOPEDOT:PSS/SPCEs also have the lowest charge transfer resistance $\left(\mathrm{R}_{\mathrm{ct}}\right)$ of $163.82 \Omega$.

However, the non-directed immobilization of enzymes on prGO-PEDOT:PSS/SPCEs could compromise enzymatic conformation, thus reducing GOx catalytic activity. This can be seen when GOx/SPCEs, GOx/GO-PEDOT:PSS/SPCEs , and GOx/prGO-PEDOT:PSS/SPCEs were tested in glucose concentration of 0.2, 0.6 and $1 \mathrm{mM}$; the least obvious change in peak current $\left(\mathrm{I}_{\mathrm{p}}\right)$ was seen for GOx/prGO-PEDOT:PSS/SPCEs, and the most obvious change in $\mathrm{I}_{\mathrm{p}}$ for GOx/GO/SPCEs . Although prGO-PEDOT:PSS/SPCEs have a larger surface area involved in the redox reaction in $\mathrm{K}_{3}\left[\mathrm{Fe}(\mathrm{CN})_{6}\right]$, and that increase of $R_{c t}$ suggests immobilization of enzymes, GOx/prGO-PEDOT:PSS/SPCEs could not differentiate between the low, intermediate, and high glucose concentrations, where there is no obvious change in $\mathrm{I}_{\mathrm{p}}$. The results could be due to the $\mathrm{GO}_{\mathrm{x}}$ structure when immobilized on electrode surfaces, which could impede enzyme catalytic activity.

Future studies will focus on increasing the changes in $\mathrm{I}_{\mathrm{p}}$ for all electrode types at different glucose concentrations through optimizing enzyme immobilization on different materials and statistical tools such as machine learning to better understand the effect of materials on $\mathrm{I}_{\mathrm{p}}$.

\section{Acknowledgment}

The research work is funded by the Malaysia Ministry of Education, Fundamental Research Grant Scheme (FRGS17 037-0603) awarded to Dr. Wan Wardatul Amani Wan Salim and the IIUM Research Postdoctoral Fellowship Scheme (RPDF18-002-0002).

\section{References}

[1] Wisitsoraat, A., Pakapongpan, S., Sriprachuabwong, C., Phokharatkul, D., Sritongkham, P., Lomas, T. \& Tuantranont, A. (2013). Graphene-PEDOT:PSS on screen printed carbon electrode for enzymatic biosensing. Journal of Electroanalytical Chemistry, 704, 208-213.

[2] Sriprachuabwong, C., Karuwan, C., Wisitsorrat, A., Phokharatkul, D., Lomas, T., Sritongkham, P. \& Tuantranont, A. (2012). Inkjet-printed graphene-PEDOT:PSS modified screen printed carbon electrode for biochemical sensing. Journal of Materials Chemistry, 22, 5478-5485.

[3] Lawal, A. T. (2015). Synthesis and utilis ation of graphene for fabrication of electrochemical sensors. Talanta, 131, 424-443.

[4] Lee, S. B., Lee, S. M., Park, N. Il, Lee, S. \& Chung, D. W. (2015). Preparation and characterization of conducting polymer nanocomposite with partially reduced graphene oxide. Synthetic Metals, 201, 61-66.

[5] Raj, P. G., Rani, V. S., Kanwat, A. \& Jang, J. (2016). Enhanced organic photovoltaic properties via structural modifications in PEDOT:PSS due to graphene oxide doping. Materials Research Bulletin. 74, 346-352.

[6] Niu, J., Yang, D., Ren, X., Yang, Z., Liu, Y., Zhu, X., Zhao, W. \& Liu, S. (2017). Graphene-oxide doped PEDOT:PSS as a superior hole transport material for high-efficiency perovskite solar cell. Organic Electronics: Physics, Materials, Applications. 48, 165-171.

[7] Hong, W., Xu, Y., Lu, G., Li, C. \& Shi, G. (2008). Transparent graphene/PEDOT-PSS composite films as counter electrodes of dye-sensitized solar cells. Electrochemistry Communications. 10, 1555-1558.

[8] Zhang, H., Xu, J., Wen, Y., Wang, Z. \& Zhang, J. (2015). Conducting poly(3,4ethylenedioxythiophene):poly(styrene- sulfonate) film electrode with superior long-term electrode stability in water and synergistically enhanced electrocatalytic ability for application in electrochemical sensors. Synthetic Metals. 204, 39-47. 
[9] Liu, Y., Weng, B., Razal, J. M., Xu, Q., Zhao, C., Hou, Y., Seyedin, S., Jalili, R., Wallace, G. G. \& Chen, J. (2015). High-performance flexible all-solid-state supercapacitor from large free-standing graphene-PEDOT/PSS films. Scientific Report, 5, 1-11.

[10] Lee, J.T., Choi, S.H. \& Kim, J.Y. (2014). Electrical conductivity of poly(3,4-ethylenedioxythiophene):p-toluene sulfonate films hybridized with reduced graphene oxide. Nanoscale Research Letter. 9, 1-10.

[11] Yang, W., Zhao, Y., He, X., Chen, Y., Xu, J., Li, S., Yang, Y. \& Jiang, Y. (2015). Flexible conducting polymer/reduced graphene oxide films: synthesis, characterization, and electrochemical performance. Nanoscale Research Letter, 10, 1-7.

[12] Kim, H., Lee, J., Ok, S. and Choe, Y. (2012). Effects of pentacene-doped PEDOT : PSS as a hole- conducting layer on the performance characteristics of polymer photov oltaic cells. Nanoscale Research Letter, 7, 1-8.

[13] Montes-Navajas, P., Asenjo, N. G., Santamaría, R., Menéndez, R., Corma, A. \& García, H. (2013). Surface area measurement of graphene oxide in aqueous solutions. Langmuir, 29, 13443-13448.

[14] Warner, J. H., Schaffel, F., Rummeli, M. \& Bachmatiuk, A. (2013). Graphene : Fundamentals and Emergent Applications (1st ed.), London, UK: Elsevier Inc.

[15] Pumera, M. (2011). Graphene in biosensing. Materials Today, 14, 308-315.

[16] Park, C. S., Lee, C. \& Kwon, O. S. (2016). Conducting polymer based nanobiosensors. Polymers, 8, 1-18.

[17] Tehrani, F., Reiner, L. \& Bavarian, B. (2015). Rapid prototyping of a high sensitivity graphene based glucose sensor strip. PLoS One, 10, 1-11.

[18] Hu, L., Li, M., Yang, K., Xiong, Z., Yang, B., Wang, M., Tang, X., Zang, Z., Liu, X., Li, B., et al. (2018) . PEDOT:PSS monolayers to enhance the hole extraction and stability of perovskite solar cells. Journal of Materials Chemistry A, 6, 16583-16589.

[19] Jäckle, S., Liebhaber, M., Gersmann, C., Mews, M., Jäger, K., Christiansen, S. \& Lips, K. (2017). Potential of PEDOT:PSS as a hole selective front contact for silicon heterojunction solar cells. Scientific Report, 7, 1-8.

[20] Wu, X., Lian, L., Yang, S. \& He, G. (2016). Highly conductive PEDOT:PSS and graphene oxide hybrid film from a dipping treatment with hydroiodic acid for organic light emitting diodes. Journal of Materials Chemistry C, 4, $8528-8534$.

[21] Wang, Y. H., Deng, H. H., Liu, Y. H., Shi, X. Q., Liu, A. L., Peng, H. P., Hong, G. L. \& Chen, W. (2016). Partially reduced graphene oxide as highly efficient DNA nanoprobe. Biosensors Bioelectronics, 80, 140-145.

[22] Zhu, X., Xu, J., Duan, X., Lu, L., Zhang, K., Yu, Y., Xing, H., Gao, Y., Dong, L., Sun, H., et al. (2015). Controlled synthesis of partially reduced graphene oxide: Enhance electrochemical determination of isoniazid with high sensitivity and stability. Journal of Electroanalytical Chemistry, 757, 183-191.

[23] Zhang, Z. \& Yin, J. (2014). Sensitive detection of uric acid on partially electro-reduced graphene oxide modified electrodes. Electrochimica Acta, 119, 32-37.

[24] Jiang, Y., Hu, C., Cheng, H., Li, C., Xu, T., Zhao, Y., Shao, H. \& Qu, L. (2016). Spontaneous, straightforward fabrication of partially reduced graphene oxide-polypyrrole composite films for versatile actuators. ACS Nano, $10,4735-4741$.

[25] Nirala, N. R., Abraham, S., Kumar, V., Pandey, S. A., Yadav, U., Srivastava, M., Srivastava, S. K., Singh, V. N., Kayastha, A. M., Srivastava, A., et al. (2015). Partially reduced graphene oxide-gold nanorods composite based bioelectrode of improved sensing performance. Talanta, 144, 745-754.

[26] Pei, S. \& Cheng, H. M. (2012). The reduction of graphene oxide. Carbon, 50, 3210-3228.

[27] Ramli, N. I., Ismail, N. A. B., Abd-Wahab, F. \& Wan Salim, W. W. A. (2018). Cyclic voltammetry and electrical impedance spectroscopy of electrodes modified with PEDOT:PSS-reduced graphene oxide composite. In Transparent Conducting Films, (pp 1-10). United Kingdom:IntechOpen.

[28] Ismail, N. A. B., Abd-Wahab, F., Ramli, N. I., Bader, M. M. \& Wan Salim, W. W. A. (2019). Electrochemical methods to characterize nanomaterial-based transducers for the Development of Noninvasive Glucose Sensors. In Siddiquee, S., Melvin, G. J. H., \& Rahman, M. M., Nanotechnology: Applications in Energy, Drug and Food, (pp 423-439), Switzerland: Springer International Publishing.

[29] Unnikrishnan, B., Palanis amy, S. \& Chen, S. M. (2013). A simple electrochemical approach to fabricate a glucose biosensorbased on graphene - glucose oxidase biocomposite. Biosensors Bioelectronics, 39, 70-75.

[30] Guthoos, H. F. A., Noorin, N. N., Ismail, N. A. B., Tumian, A. \& Wan Salim, W. W. A. (2018). Effect of gold nanoparticles and electrode drying time on reduced graphene oxide-based composite with respect to peak current of cyclic voltammetry. ARPN Journal of Engineering Applied Sciences, 13, 1420-1426.

[31] Ismail, N. A. B., Arris, F. A., Tumian, A. and Wan Salim, W. W. A. (2019). Preliminary study on the effect of reduced graphene oxide, gold nanoparticles, and Nafion ${ }^{\circledR}$ concentration on redox peak current for electrochemical biosensing. Journal of Engineering, Science and Technology, 14, 47-57.

[32] Rafighi, P., Tavahodi, M. \& Haghighi, B. (2016). Fabrication of a third-generation glucose biosensor using graphene-polyethyleneimine-gold nanoparticles hybrid. Sensors and Actuators, B: Chemical, 232, 454-461.

[33] Jugović, B., Grgur, B., Antov, M., Knežević-Jugović, Z., Stevanović, J. \& Gvozdenović, M. (2016). Polypyrrolebased enzyme electrode with immobilized glucose oxidase for electrochemical determination of glucose. International Journal of Electrochemical Science, 11, 1152-1161. 
[34] House, J. L., Anderson, E. M. \& Ward, W. K. (2007). Immobilization techniques to avoid enzyme loss from oxidase-based biosensors: A one-year study. Journal of Diabetes Science and Technology, 1, 18-27.

[35] Shi, J., Claussen, J. C., McLamore, E. S., Ul Haque, A., Jaroch, D., Diggs, A. R., Calvo-Marzal, P., Rickus, J. L. \& Porterfield, D. M. (2011). A comparative study of enzyme immobilization strategies for multi-walled carbon nanotube glucose biosensors. Nanotechnology 22, 1-10.

[36] Migneault, I., Dartiguenave, C., Bertrand, M. J. \& Waldron, K. C. (2004). Glutaraldehyde: Behavior in aqueous solution, reaction with proteins, and application to enzyme crosslinking. Biotechniques, 37, 790-802.

[37] Barbosa, O., Ortiz, C., Berenguer-Murcia, Á., Torres, R., Rodrigues, R. C. \& Fernandez-Lafuente, R. (2014). Glutaraldehyde in bio-catalysts design: a useful crosslinker and a versatile tool in enzyme immobilization. RSC Advances, 4, 1583-1600.

[38] Wang, J., Pedrero, M., Sakslund, H. \& Hammerich, O. (1996). Electrochemical activation of screen-printed carbon strips. Analyst 121, 345-350.

[39] González-Sánchez, M. I., Gómez-Monedero, B., Agrisuelas, J., Iniesta, J. \& Valero, E. (2018). Highly activated screen-printed carbon electrodes by electrochemical treatment with hydrogen peroxide. Electrochemistry Communications, 91, 36-40.

[40] Ismail, N. A. B., Abd-Wahab, F. \& Wan Salim, W. W. A. (2018). Cyclic voltammetry and electrochemical impedance spectroscopy of partially reduced graphene oxide - PEDOT:PSS transducer for biochemical sensing, 2018 IEEE-EMBS Conference on Biomedical Engineering and Sciences (IECBES). Sarawak, Malaysia.

[41] Abd-Wahab, F., AbdulGuthoos, H. F. \& Wan Salim, W.W. A. (2019). Solid-state rGO-PEDOT:PSS transducing material for cost-effective enzymatic sensing. Biosensors, Multidisciplinary Digital Publishing Institute 9, 1-15.

[41] Lindfors, T., Österholm, A., Kauppila, J. \& Pesonen, M. (2013). Electrochemical reduction of graphene oxide in electrically conducting poly(3,4-ethylenedioxythiophene) composite films. Electrochimica Acta, 110, 428-436.

[42] Elgrishi, N., Rountree, K. J., McCarthy, B. D., Rountree, E. S., Eisenhart, T. T. \& Dempsey, J. L. (2018). A practical beginner's guide to cyclic voltammetry. Journal of Chemical Education, 95, 197-206.

[43] Benoudjit, A., Bader, M. M., \& Wan Salim, W. W. A. (2018). Study of electropolymerized PEDOT:PSS transducers for application as electrochemical sensors in aqueous media. Sensing and Bio-Sensing Research, 17, $18-24$.

[44] Randviir, E. P. \& Banks, C. E. (2013). Electrochemical impedance spectroscopy: an overview of bioanalytical applications. Analytical Methods, 5, 1098-1115.

[45] Orazem, M. E. \& Tribollet, B. (2008). Electrochemical Impedance Spectroscopy (1st ed.), Hoboken New Jersey, USA: John Wiley \& Sons Inc.

[46] Lisdat, F. \& Schäfer, D. (2008). The use of electrochemical impedance spectroscopy for biosensing. Analytical and Bioanalytical Chemistry, 391, 1555-1567.

[47] Wang, J. (2006). Analytical Electrochemistry (3rd ed.), Hoboken New Jersey, USA: John Wiley \& Sons Inc.

[48] Fernández-Sánchez, C., McNeil, C. J. \& Rawson, K. (2005) Electrochemical impedance spectroscopy studies of polymer degradation: Application to biosensordevelopment. TrAC - Trends in Analytical Chemistry, 24, 37-48.

[49] Santos, A., Davis, J. J. \& Bueno, P. R. (2014). Fundamentals and applications of impedimetric and redox capacitive biosensors. Journal of Analytical \& Bioanalytical Techniques, 7, 1-15.

[50] Pänke, O., Balkenhohl, T., Kafka, J., Schäfer, D. \& Lisdat, F. (2007). Impedance spectroscopy and biosensing. Advances in Biochemical Engineering/Biotechnology, 109, 195-237.

[51] Rivnay, J., Inal, S., Collins, B. A., Sessolo, M., Stavrinidou, E., Strakosas, X., Tassone, C., Delongchamp, D. M. and Malliaras, G. G. (2016). Structural control of mixed ionic and electronic transport in conducting polymers. Nature Communications, 7, 1-9.

[52] Santhosh,P., Manesh, K. M., Uthayakumar, S., Komathi, S., Gopalan, A. I. \& Lee, K. P. (2009). Fabrication of enzymatic glucose biosensorbased on palladium nanoparticles dispersed onto poly(3,4-ethylenedioxythiophene) nanofibers. Bioelectrochemistry, 75, 61-66.

[53] De Taxis Du Poet, P., Miyamoto, S., Murakami, T., Kimura, J. \& Karube, I. (1990). Direct electron transfer with glucose oxidase immobilized in an electropolymerized poly( $\mathrm{N}$-methylpyrrole) film on a gold microelectrode. Analytica Chimica Acta, 235, 255-263.

[54] Kumar, S. D., Kulkarni, A. V., Dhaneshwar, R. G. \& D’Souza, S. F. (1992). Cyclic voltammetric studies at the glucose oxidase enzyme electrode. Journal of Electroanalytical Chemistry, 342, 153-160.

[55] Andreasen, J. W., Mitraka, E., Berggren, M., Crispin, X., Tybrandt, K., Zozoulenko, I. V., Zhao, D., Volkov, A. V., Wijeratne, K. \& Ail, U. (2017). Understanding the capacitance of PEDOT:PSS. Advanced Functional Materials, 27, 1-18.

[56] Nguyen Huynh, N. M., Boeva, Z. A., Smått, J. H., Pesonen, M. \& Lindfors, T. (2018). Reduced graphene oxide as a water, carbon dioxide and oxygen barrier in plasticized poly(vinyl chloride) films. RSC Advances, 8, 1764517655 . 